\title{
La gestión territorial requiere coherencia. Una comprobación en Colombia
}

\section{Territorial management requires coherence. A check in Colombia}

\author{
Adriana Posada-Arrubla ${ }^{1}$ \\ ${ }^{1}$ Economista Agrícola, M.Sc. Universidad de Ciencias Aplicadas y Ambientales U.D.C.A, Programa de Ingeniería Geográfica. Bogotá D.C., Colombia; e-mail: \\ aposada@udca.edu.co; (D) https://orcid.org/0000-0002-2440-0805
}

Cómo citar: Posada-Arrubla, A. 2020. La gestión territorial requiere coherencia. Una comprobación en Colombia. Rev. U.D.C.A Act. \& Div. Cient. 23(2):e1599. http://doi.org/10.31910/rudca.v23.n2.2020.1599

Artículo de acceso abierto publicado por Revista U.D.C.A Actualidad \& Divulgación Científica, bajo una licencia Creative Commons CC BY-NC 4.0

Publicación oficial de la Universidad de Ciencias Aplicadas y Ambientales U.D.C.A, Institución de Educación Superior Acreditada de Alta Calidad por el Ministerio de Educación Nacional.

Recibido: Junio 12 de 2020 Aceptado: Diciembre 3 de 2020 Editado por: Ingeborg Zenner de Polanía

\section{RESUMEN}

Existe coherencia en la gestión territorial si, de manera gradual y en el largo plazo, se evidencia que los proyectos planificados en conjunto con la comunidad, efectivamente, se realizan con calidad y ajustados a cambios exigidos en la relación sociedad-naturaleza. El objetivo de esta investigación fue identificar distintos aspectos para evaluar la coherencia de la gestión territorial y probar algunos de ellos en Colombia. El estudio, se llevó a cabo en cuatro etapas: análisis de la gestión territorial desde la coherencia; análisis de los instrumentos de planeación territorial, en los cuatro principales municipios del país; contraste de datos financieros en el componente ambiental y aplicación de entrevistas a personas representativas de la comunidad. Se logró plantear: tres categorías con 13 parámetros, para la medición de la gestión territorial; el análisis de 24 instrumentos locales en Barranquilla, Bogotá, Medellín y Cali; se profundizó en la forma en que el componente ambiental es preponderante en la gestión territorial y se evidenció con la comunidad, la distancia existente entre el desarrollo planeado y los proyectos realizados. El estudio muestra la urgencia en la transformación de las acciones sobre el territorio local, de manera colectiva, continua, consistente, con calidad y con visión ambiental y global.
Palabras clave: Gobernanza; Políticas internas; Planeación; Geografía aplicada; Colombia.

\section{ABSTRACT}

This research was carried out to identify different aspects to evaluate the coherence of territorial management and, to prove some of them, to the interior of Colombia. The methodology includes: analysis of territorial management from the coherence approach; analysis of the instruments of territorial planning, in the four main municipalities; contrast of financial data on the environmental component; and, application of interviews to representative people of the community, to verify results. It was possible to put forward three categories with 13 parameters, for the measurement of territorial management; the analysis of 24 local instruments. The way in which the environmental component plays a preponderant role for territorial management was deepened; and, it was evidenced with the community, the distance between the planned development and the projects accomplished. The study shows the urgency in the transformation of actions on the local territory, collectively, continuously, consistently, with quality and environmental and global vision. 
Keywords: Government; Internal politics; Planning; Applied geography; Colombia.

\section{INTRODUCCIÓN}

El enfoque de esta investigación es el desarrollo territorial visto como macroproceso y en su interior, se consideran procesos, como planeación, ordenamiento y gestión. A su vez, los procesos contienen componentes, como el ambiental. En Colombia, por una parte, la planeación del desarrollo aún es concebida como un asunto de corto plazo, trabaja temas sectoriales y administra el presupuesto público, mientras que, por otra parte, el ordenamiento territorial, se traza a largo plazo, con un esquema técnico, que incluye prospectiva y cartografía y poca integración al presupuesto público. El abordaje de la gestión territorial aún no se concibe como una parte importante del macroproceso de desarrollo territorial. Permanece latente la duda entre lo que se planifica y lo que realmente se hace y el factor común, de la mayoría de los planes, está en el vacío de las acciones propuestas. Por ello, la acción es la lupa puesta en esta investigación.

La gestión, según el sujeto sobre el cual recae la acción o el objetivo a lograr, puede ser de tipo empresarial, pública, social, ambiental o territorial. Las diferencias están en la forma de medir avances y en el uso de instrumentos específicos. La gestión territorial, por ser un proceso complejo, debe abordar asuntos dinámicos y abiertos de tipo no lineal y no cerrado. Siguiendo a Arango Fernández (2007), para poder gestionar un sistema complejo es necesario conocer los fundamentos, que dan lugar a esa complejidad.

La gestión territorial es interpretada de diversas formas, como la ingeniería de las intervenciones territoriales (Boisier, 2001); un método para hacer intervención en el territorio (González Fuenzalida, 2011); el cumplimiento gradual y ajustado de políticas públicas de largo plazo, con la lógica de gestión por proyectos para el desarrollo (Rivera Pabón, 2013); la concertación entre entidades territoriales para crear territorios gobernables (Espinosa Cuervo, 2006); un modelo emergente y aún limitado (Dalla-Torre, 2017); un conjunto de acciones institucionales sobre el territorio (López Trigal, 2015) y de una manera contundente y especial, en el marco de la Unión Europea, Farinós Dasí (2008) relaciona el principio de la coherencia con el de gobernanza territorial; sin embargo, se aclara que, no se trabaja la cohesión territorial, ya que es un término utilizado en la Unión Europea (Gareth, 2014) y a que, en América Latina, aún no se incorpora en la comprensión del desarrollo territorial (Mondragón Rivera, 2018), con excepción del acercamiento realizado por Cabeza-Morales (2015) y los llamados de la CEPAL (2010), para mejorar la eficiencia en la administración territorial de esta región.

En América Latina, García \& García (2010) analizaron la gestión para resultados del desarrollo, en 25 países, aplicando siete indicadores, para establecer que cada país posee debilidades y fortalezas particulares, pero en todos es imperante conciliar lo urgente con lo posible y lo deseado. Morea (2020), al analizar los modelos de desarrollo y soberanía de la región, evidencia la necesidad de una visión largoplacista y de la integración, para tratar desequilibrios territoriales, problemas ambientales y conflictos sociales.
En Colombia, desde 1991, la Constitución Política establece que se debe avanzar hacia el desarrollo territorial, con metodologías y normas sobre planificación territorial, pero no especifica sobre gestión territorial, a excepción de la gestión ambiental urbana, impulsada por el MAVDT (2008). En este país, Posada (2014) comparó los principales municipios, encontrando que, a pesar de las diferencias, hay luces para mejorar el modelo de desarrollo territorial con el componente ambiental, como eje articulador. Otro estudio similar planteó una propuesta, a nivel departamental, bajo el enfoque de la cohesión (Sánchez-Zamora et al. 2017), pero específicamente, no se ha analizado la coherencia de la gestión territorial en Colombia, para determinar estrategias conducentes hacia el desarrollo territorial.

Por lo anterior, el objetivo de este artículo es identificar distintos aspectos para evaluar la coherencia de la gestión territorial y probar algunos de ellos en Colombia, haciendo énfasis en el componente ambiental.

\section{MATERIALES Y MÉTODOS}

Esta investigación, se llevó a cabo durante dos años, en cuatro etapas consecutivas, desarrolladas de la siguiente manera:

Primera etapa: Definiendo una posición para interpretar la coherencia de la gestión territorial. Se recurrió a la búsqueda de enfoques sobre coherencia, desde disciplinas distintas: filosofía (Mosterín, 2003; Molina Ochoa, 2010); física (Cortes-Tamayo, 1992; Castañeda, 2013); lingüística (Vilarnovo, 1990; Domínguez, 2009); derecho (Rodilla, 2009); psicología (Palacios-Espinosa \& RestrepoEspinosa, 2008); ecología (Gómez Sal, 2007); administración (Galán-Zazo \& Sánchez-Bueno, 2004) y política pública (Millán Acevedo, 2014). A partir de una integración conceptual es que se plantea evaluar la coherencia de la gestión territorial, mediante tres categorías, con sus respectivos indicadores.

Segunda etapa: Análisis de aspectos sobre gestión territorial, en los cuatro principales entes territoriales de Colombia: Barranquilla, Bogotá, Cali y Medellín. Colombia tiene 4 ciudades principales capitales, de 4 entes territoriales, sobre las cuales, se analizaron tres aspectos clave, contenidos en seis instrumentos de planeación territorial vigentes para el 2018: temporalidad, escalaridad y objetivos. En el cuadro 1, se sintetizan los 24 instrumentos estudiados, con la siguiente tipología: dos locales: plan de desarrollo (PD) y plan de ordenamiento territorial (POT); dos regionales: plan de acción ambiental (PAA) y el plan de gestión ambiental regional (PGAR); uno departamental: el plan de desarrollo (PDD); uno estratégico, que cuantifican en costos los proyectos prioritarios para el desarrollo territorial, conjugando al PD y, al POT: el programa de ejecución (PE).

Tercera etapa: Búsqueda de la coherencia en la gestión territorial, a través del componente ambiental. Se realizaron dos tipos de búsqueda: 
Cuadro 1. Instrumentos de planeación territorial utilizados en los principales Municipios de Colombia, desde el 2016.

\begin{tabular}{|c|c|c|c|c|}
\hline $\begin{array}{l}\text { INSTRUMENTOS } \\
\text { DESARROLLO } \\
\text { TERRITORIAL }\end{array}$ & BARRANQUILLA & BOGOTÁ & CALI & MEDELLÍN \\
\hline $\begin{array}{l}\text { Plan de desarrollo 2016- } \\
2019\end{array}$ & $\begin{array}{l}\text { Barranquilla "Capital de } \\
\text { vida" }\end{array}$ & $\begin{array}{l}\text { Bogotá "mejor para } \\
\text { todos" }\end{array}$ & Cali "progresa contigo" & $\begin{array}{l}\text { Medellín “cuenta con } \\
\text { vos" }\end{array}$ \\
\hline $\begin{array}{l}\text { Plan de ordenamiento } \\
\text { territorial }\end{array}$ & $\begin{array}{l}\text { Decreto } 0212 \text { de } 2014 \text {. } \\
\text { Se adopta la revisión } \\
\text { y ajuste de largo plazo } \\
\text { 2012- } 2032 \text {. }\end{array}$ & $\begin{array}{l}\text { Decreto } 364 \text { de } \\
\text { 2013. Modificación } \\
\text { excepcional de las } \\
\text { normas urbanística. } \\
\text { Suspendido por } \\
\text { Comisión } 1^{\text {a }} \text {. Consejo } \\
\text { Estado, } 2014 \text {. }\end{array}$ & $\begin{array}{l}\text { Acuerdo } 0373 \text { de } 2014 . \\
\text { Revisión ordinaria de } \\
\text { largo plazo. 2014-2027 }\end{array}$ & $\begin{array}{l}\text { Acuerdo } 048 \text { de } 2014 . \\
\text { Se adopta la revisión y } \\
\text { ajuste de largo plazo. } \\
\text { 2014-2027 }\end{array}$ \\
\hline Programa de ejecución & $\begin{array}{l}\text { Explícito: Planteado en } \\
\text { POT y vinculado en PD. }\end{array}$ & $\begin{array}{l}\text { Implícito: Suspendido, } \\
\text { pero con análisis. }\end{array}$ & $\begin{array}{l}\text { Explícito: Planteado en } \\
\text { POT y vinculado en PD. }\end{array}$ & $\begin{array}{l}\text { Explícito: Planteado en } \\
\text { POT y vinculado en PD. }\end{array}$ \\
\hline $\begin{array}{l}\text { Plan de desarrollo } \\
\text { departamental 2016- } \\
2019\end{array}$ & Atlántico líder & $\begin{array}{l}\text { Cundinamarca unidos } \\
\text { podemos más }\end{array}$ & El Valle está en vos & $\begin{array}{l}\text { Antioquia piensa en } \\
\text { grande }\end{array}$ \\
\hline $\begin{array}{l}\text { Plan de gestión } \\
\text { ambiental regional }\end{array}$ & $\begin{array}{l}\text { CRAUTÓNOMA 2012- } \\
2022\end{array}$ & $\begin{array}{l}\text { CAR 2012-2023 } \\
\text { (Departamentos de } \\
\text { Boyacá y Cundinamarca) }\end{array}$ & CVC 2015-2036 & $\begin{array}{l}\text { CORANTIOQUIA } \\
2007-2019 \\
\text { (+ Cornare }+ \\
\text { Corpourabá) }\end{array}$ \\
\hline $\begin{array}{l}\text { Plan de acción ambiental } \\
\text { 2016-2019 }\end{array}$ & CRAUTÓNOMA & $\begin{array}{l}\text { CAR (Departamentos de } \\
\text { Boyacá y Cundinamarca) }\end{array}$ & $\begin{array}{l}\text { En construcción } \\
\text { concertada }\end{array}$ & $\begin{array}{l}\text { CORANTIOQUIA } \\
2007-2019 \\
\text { (+ Cornare }+ \\
\text { Corpourabá) }\end{array}$ \\
\hline
\end{tabular}

Propuestas referidas al componente ambiental: Análisis vertical y horizontal en los diferentes instrumentos de planeación, para establecer las formas de ir modelando el territorio, con la inclusión del componente ambiental.

Análisis financiero con énfasis en el componente ambiental. Con los datos del sistema de control de finanzas públicas (SISFUTDNP), correspondientes a 2016 del instrumento plan de desarrollo, en cuatro tipos de aspectos púbicos: a) Agua y saneamiento; b) Agrícola; c) Naturaleza; d) Gestión del riesgo. Se comparó el dinero presupuestado, contra el invertido; se calculó un porcentaje de ejecución del gasto y el promedio de su cumplimiento.

Cuarta etapa: Visión de la comunidad sobre la gestión territorial. Directamente en Barranquilla, Bogotá, Cali y Medellín, se contactaron a más de 30 personas, entre funcionarios públicos y representantes de la comunidad, quienes han desempeñado actividades de gestión territorial, se les aplicó una entrevista presencial semiestructurada, con 15 preguntas, entre las que se destacan: ¿qué entiende por gestión territorial? ¿Por qué el vacío entre lo planificado y lo que se ha debido hacer? ¿Cuáles pueden ser las mejores alianzas para generan funcionalidad territorial? ¿Cómo se imagina a futuro este municipio? ¿Qué congestiona o impide el desarrollo territorial?

\section{RESULTADOS Y DISCUSIÓN}

Interpretación y parámetros de medición para la coherencia de la gestión territorial. Coherencia, viene del latín cohaerentia y se compone de con/co (conjuntamente, globalmente), más la raíz del verbo haerere (estar pegado, estar unido, estar vinculado), con el sufijo etnia que indica la cualidad de un agente (Diccionario Etimológico de Chile, 2015). Según la RAE (2014) existen dos significados de coherencia: de una parte, es conexión, relación o unión de unas cosas con otras y, de otra, es la actitud lógica y consecuente con una posición anterior. Al seleccionar 8 enfoques, sintetizados en el cuadro 2, en esta investigación, se pudo definir que la gestión territorial es: una actitud coherente sobre un territorio que corresponde a un proceso dinámico, capaz de aglutinar la multitemporalidad y la multiescalaridad, desde proyectos estratégicos locales, permitiendo la cooperación funcional entre actores, para obtener resultados que puedan estructurar, gradualmente, la realidad de un modelo de desarrollo territorial. A partir de esta definición, se plantea una forma, que se podría utilizar para medir y comparar la gestión territorial, al interior de un país o entre países, con características comunes. 
Cuadro 2. Compilación de enfoques sobre la coherencia desde diferentes disciplinas.

\begin{tabular}{|l|l|}
\hline \multicolumn{1}{|c|}{ DISCIPLINA } & \multicolumn{1}{c|}{ ENFOQUE } \\
\hline 1. Filosofía & $\begin{array}{l}\text { Acciones en circunstancias sociales, que se cumplen en diversos “campos”. Es un fenómeno más dinámico, } \\
\text { que estructural, porque es cíclico. }\end{array}$ \\
\hline 2. Física & $\begin{array}{l}\text { Coherencia temporal: de un punto en dos instantes de tiempo diferentes. } \\
\text { Coherencia espacial: entre puntos distintos de una sección transversal de un haz luminoso. } \\
\text { Coherencia cuántica: que mantiene su fase en un periodo de tiempo. } \\
\text { Coherencia óptica: capacidad de las ondas luminosas superpuestas para interferir. }\end{array}$ \\
\hline 3. Lingǘ́stica & Conexión de las partes en un todo, para interpretar un texto, en torno a un tema común. \\
\hline 4. Derecho & Sistema de normas relacionadas entre sí, como parte de un conjunto de principios o valores relevantes. \\
\hline 5. Psicología & $\begin{array}{l}\text { Es la salud mental, para tener una disposición estable, que le permita a una persona, manejar las } \\
\text { circunstancias de la vida. }\end{array}$ \\
\hline 6. Ecología & Uso de los recursos naturales en función de la aptitud de los ecosistemas para soportarlos. \\
\hline 7. Administración & Relación entre la estrategia corporativa y la estructura administrativa de la organización. \\
\hline 8. Política pública & Adaptación a los procesos de cooperación, interdependencia y transnacionalización. \\
\hline
\end{tabular}

Se agruparon tres categorías, con 13 parámetros:

Categoría sociedad. Tres parámetros que se evalúan por año:

- Número de acciones de grupos, con visión de largo plazo, por año.

- Número reuniones efectivas, por año del Consejo Territorial de planeación local.

- Número de organizaciones de participación procesos de desarrollo territorial, activas por año.

Categoría planeación territorial integral. Seis parámetros a evaluar por período de gobierno local o por año, según la posibilidad de los datos:

- Porcentaje de destinación a asuntos de futuro del TOP, durante el periodo de corto plazo del DP.

- Porcentaje de destinación en el componente ambiental, en el DP.

- Porcentaje de destinación a asuntos regionales, en el DP.

- Porcentaje de destinación a asuntos internacionales, en el DP.

- Número de proyectos de inversión planteados en DP.

- Número de proyectos planteados en TOP.

Categoría ejecución recursos financieros. Cuatro parámetros a evaluar por período de gobierno local o por año, según la posibilidad de los datos:

- Presupuesto de inversión en el periodo del DP, por persona.

- Presupuesto de inversión durante el periodo del DP, por área.

- Inversión en dólares por área $\left(\mathrm{km}^{2}\right)$ y por persona, del componente ambiental.

- Porcentaje de destinación presupuestal al Programa estratégico de Ejecución, durante el periodo del DP.

Aspectos de gestión territorial, en los cuatro principales entes territoriales de Colombia.

Se destacan elementos sobre tres aspectos: los objetivos generales, la escala y el tiempo, así:
LOS OBJETIVOS: Solo 2 POT se han aprobado concertadamente con el Concejo Municipal: Cali y Medellín. La importancia del territorio rural no es relevante ni explícita. El tema recurrente es gestión del riesgo. Las propuestas sobre ciudad tienen relación directa con el concepto de calidad del agua y del aire. La estructura ecológica principal, ecosistemas estratégicos y temas subregionales, presentan particularidades por caso. Solo Medellín planteó la categoría de suelo suburbano. Todos destacan su importante ubicación estratégica: Bogotá y Medellín, en el centro; Barranquilla, en el mar Caribe y Cali, en el océano Pacífico. Solo en el 25\% de las propuestas, se hacen planteamientos presupuestales explícitos y detallados.

LA ESCALARIDAD: Heterogénea en todos los casos, pues en los objetivos generales de los instrumentos, se intenta enfocar el desarrollo dentro del gran sistema territorial, pero en los proyectos concretos, se le otorga énfasis a la acción en la ciudad, como si fuera un espacio aparte de la ruralidad, de la cuenca o de importantes ecosistemas. Se encuentra el enfoque de ciudad-región o ciudaddepartamento y de relaciones globales.

LA TEMPORALIDAD: El POT de Barranquilla supera el largo plazo exigido de los 3 periodos de gobierno, extendiendo su duración por 5 periodos. El programa de ejecución muestra un buen ejercicio al plantear proyectos priorizados y cuantificar sus costos; con excepción de Bogotá, por tener suspendido su POT. En la figura 1, además de mostrar la localización de los municipios bajo análisis, se sintetizan los datos sobre la temporalidad, para comparar visiones de futuro.

\section{Coherencia en la gestión territorial, a través del componente ambiental.}

Lo que muestran las propuestas: Para Barranquilla, el cambio climático es lo más relevante y está en relación con la propuesta del Atlántico; también importa la gestión del riesgo y el manejo del río 


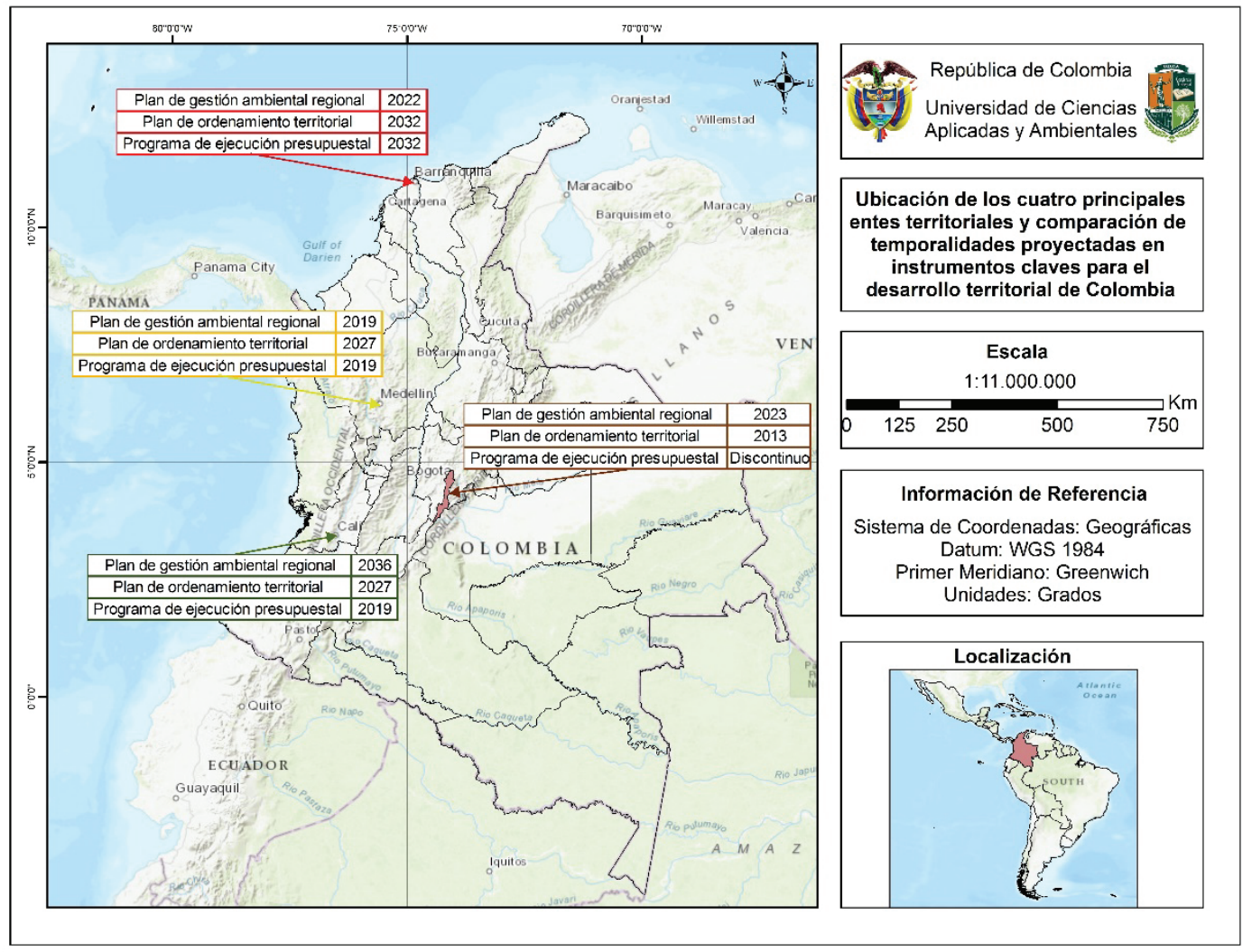

Figura 1. Ubicación de los cuatro principales entes territoriales y comparación de temporalidades proyectadas en instrumentos clave, para el desarrollo territorial Colombia.

Magdalena. Para Bogotá, la estructura ecológica principal es lo más relevante; aborda un enfoque de sostenibilidad, en relación con la propuesta de Cundinamarca y considera la gestión del riesgo y la adaptación al cambio climático. Para Cali, la gestión del riesgo es lo más relevante; aborda un enfoque de sostenibilidad, que está en relación con la propuesta del Valle del Cauca; también, incluye la calidad ambiental, la conservación ecológica, el manejo de cuencas, la cultura y la gobernanza ambiental. Para Medellín, su enfoque de sostenibilidad y de sistemas ambientales es lo más relevante y se complementa con la estructura ecológica principal, la gestión del riesgo, la adaptación al cambio climático, que está en relación con la propuesta de Antioquia y se incorporan tres tipos de gestión: ambiental, del agua, territorial.

Lo que muestran las finanzas: En la tabla 1, se destacan indicadores que pueden ser usados, para comparar la aproximación a la gestión territorial, en diferentes casos y países. En Colombia, es clara la distancia entre lo planeado y lo ejecutado; sin embargo, Barranquilla da ejemplo al realizar un mayor esfuerzo, mientras que Bogotá, siendo la capital del país, no sale a relucir. Los datos en dólares de la tabla, muestran la baja capacidad de inversión de países en vía de desarrollo y la poca importancia financiera del componente ambiental, dentro del presupuesto total para el desarrollo, dejando ver mínima visión de futuro y el escaso interés real por recuperar la base de la existencia humana. En la figura 2, se sintetiza el indicador presupuestal de ejecución en cada caso, sobre los asuntos del componente ambiental, con cifras en millones de pesos colombianos. En ninguno de los entes territoriales analizados, se cumple el gasto presupuestado al 100\%, pero, en promedio, el cumplimiento de las acciones de Barranquilla es el más alto. En Bogotá, no se considera el gasto para aspectos agrícolas, a pesar de tener un área rural destacada y el menor nivel del gasto es para gestión del riesgo, en contravía de lo que se planteó ampliamente en las propuestas escritas. En Cali, el gasto en el componente ambiental es el menor de los cuatro casos y aunque sí se considera inversión para aspectos agrícolas, es el menos representativo. En Medellín, también se considera el gasto para aspectos agrícolas.

Opiniones de la comunidad sobre la gestión territorial. Para la mayoría de los entrevistados ubicados en las 4 diferentes ciudades, desde sus diferentes ópticas como funcionarios, empresarios, líderes o académicos, el término gestión territorial es fácil de comprender; lo relacionan con el futuro y con diferentes tipos de acciones a realizar, para llegar a unos resultados de beneficio colectivo. Para varias personas, el vacío no está en la acción, sino en el control de la calidad de la acción. Se destaca, que la alianza privilegiada por los entrevistados para una adecuada gestión territorial es la unión Estado + academia + empresarios e industriales + comunidades; expresado de otra forma, corresponde a los diferentes sectores de la sociedad, que complementan los aspectos de validación social + recursos humanos y económicos + desarrolladores de metas + divulgadores 
Tabla 1. Comparación porcentual y en dólares de indicadores sobre gestión territorial, entre los cuatro principales entes territoriales de Colombia.

\begin{tabular}{|l|c|c|c|c|c|}
\hline $\begin{array}{c}\text { Ente } \\
\text { territorial }\end{array}$ & $\begin{array}{c}\text { Cumplimiento } \\
\text { inversión } \\
\text { ambiental }\end{array}$ & $\begin{array}{c}\text { Inversión } \\
\text { ambiental } \\
\text { US/año }\end{array}$ & $\begin{array}{c}\text { Inversión } \\
\text { ambiental } \\
\text { US/area } \\
\mathbf{( k m}^{\mathbf{2}} \mathbf{)}\end{array}$ & $\begin{array}{c}\text { Inversión } \\
\text { ambiental US/ } \\
\text { habitante }\end{array}$ & $\begin{array}{c}\text { \% Inversión } \\
\text { ambiental, respecto } \\
\text { al total invertido }\end{array}$ \\
\hline Barranquilla & $\mathbf{7 9}$ & 42.640 & $\mathbf{2 5 7}$ & 0,03 & 7 \\
\hline Bogotá & 60 & 383.181 & 234 & 0,05 & 11 \\
\hline Cali & 52 & 30.951 & 55 & 0,01 & 4 \\
\hline Medellín & 71 & 76.872 & 201 & 0,03 & 4 \\
\hline
\end{tabular}

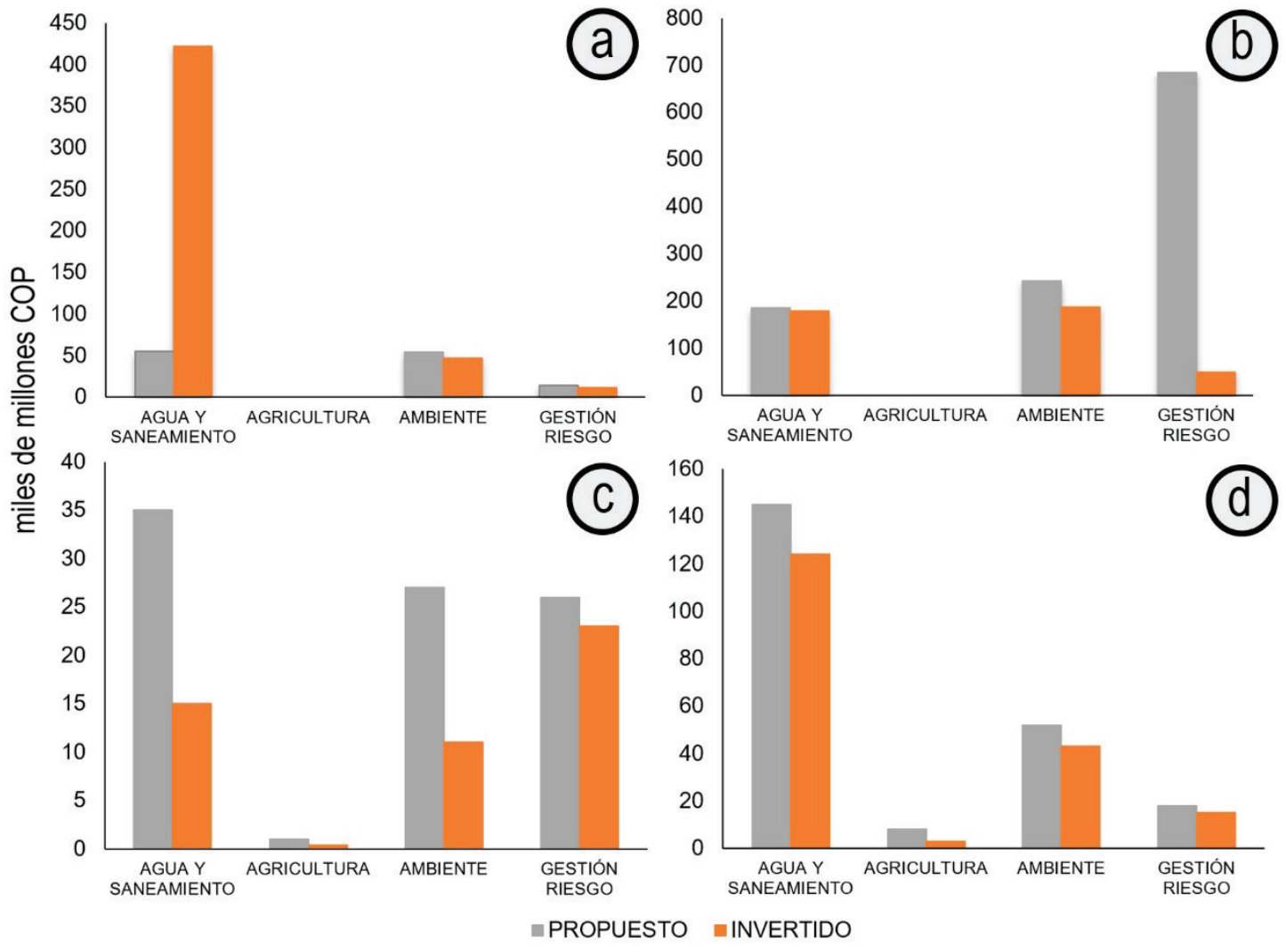

Figura 2. Comparación entre el dinero presupuestado y el invertido, en los asuntos del componente ambiental, para los cuatro principales entes territoriales de Colombia, en miles de millones COP. a. Barranquilla; b. Bogotá; c. Cali; d. Medellín.

de resultados. Algo importante es que le otorgan importancia a la cooperación bi y multilateral entre países. Respecto a la visión de futuro, los entrevistados la perciben en tres momentos: en 10 años, va existir más dinámica y más complejidad; en 20 años, las ciudades centrales de estos cuatro municipios tienden a convertirse en metrópolis, con una fuerte presión sobre las zonas rurales y los ecosistemas y en 30 años, se esperan mejoras en la educación colectiva que, a su vez, se multiplicarían en bienestar y calidad de vida, a todo nivel, es decir, que solo en el muy largo plazo, se tiene la expectativa de un cambio positivo. La congestión o los limitantes del desarrollo territorial giran en torno a diversas situaciones: la descentralización incipiente del país; la comodidad de no arriesgarse a la innovación; la debilidad en el liderazgo de los jóvenes; la falta de cultura ciudadana, de sentido de pertenencia y de pensamiento futurista; la cantidad de planes y de normas inoperantes; la debilidad en el cobro de impuestos públicos y su posterior reinversión; la competencia desleal; el narcotráfico; el conflicto armado; la desigualdad social; la falta de asociación entre municipios vecinos; la discontinuidad en los proyectos de los planes de desarrollo; los intereses políticos; y sobre todo, la corrupción, a todo nivel. 
Es para discutir, la idea que la medición de la gestión territorial no se puede hacer con elementos arbitrarios y subjetivos, sino como producto de un consenso colectivo e imparcial, pero debido a que es un asunto complejo, se requiere, mediante la coherencia, darle precisión, para establecer su alcance y su objetivo. En esta investigación, se realizó una aplicación mínima en Colombia, sobre cuatro de los 13 parámetros propuestos, pero ello no significa que se esté privilegiando el típico análisis financiero cerrado, sino motivando su integración con otros parámetros territoriales. En todo caso, posteriormente será necesario precisar la consistencia interna de las escalas de análisis, tal como lo sugieren Campo-Arias \& Oviedo (2008).

En los países de América Latina, por la complejidad política, se requiere trabajar indicadores de nivel local, con visión ambiental, regional e internacional, especialmente, porque es mucho lo que se promete en los instrumentos tipo plan y poco lo que se cumple en la realidad. Lo que se podría comprobar en Colombia, para cada uno de los 1.103 entes territoriales locales, que deben considerar, como mínimo, los seis instrumentos aquí analizados y que, como lo ha estudiado Massiris Cabezas (2012), hace parte de una circunstancia general de América Latina, en la cual, la gestión territorial es INcoherente. Lo que se une a la advertencia de Máttar \& Cuervo (2017), sobre los cinco retos para planificar el desarrollo en la región: lo multiespacial, lo multiescalar, lo intersectorial, la participación, la evaluación y el seguimiento. Se complementa la idea, con el planteamiento de Rinaudo (2004), según el cual, lo que se gestiona no es el territorio, sino el desarrollo territorial y con la precisión de Bauer \& Carrión (2016), para quienes se debe vincular al proceso, con mucha relevancia, los movimientos sociales de todo tipo.

En términos espaciales, el campo, para medir la gestión territorial, corresponde a la jurisdicción de cada ente territorial y, además, debe incluir la funcionalidad con otros entes similares, considerando a los municipios vecinos, en una visión supramunicipal, como lo sugieren Posada Arrubla et al. (2020). En términos de temporalidad, la medición de la gestión territorial, debe tener presente la gradualidad en el corto, mediano y largo plazo, considerando la dinámica de los procesos y, por ende, una evolución de la gestión territorial, que corresponda a períodos mayores a 20 años, con mínimo cinco etapas de gobierno local. En este sentido, conviene revisar la propuesta para Europa de García-Ayllón \& Miralles (2015), quienes aportan al caso, porque combina el diseño de más de 50 indicadores, con un análisis retrospectivo, usando sistemas de información geográfica, para modelar la gestión territorial a futuro.

Lo anterior significa que, en investigaciones sobre este tema, no es suficiente con evaluar comparativamente respuestas de un año respecto al siguiente, sino series de tiempo, que puedan mostrar el comportamiento de las intervenciones territoriales, bajo diferentes formas e intereses de gobierno, con lo cual, se podría establecer, si la coherencia hace parte de un cambio cultural. También, se podría evaluar lo que no se alcanzó a hacer en esta investigación, pero se propuso como la Categoría Sociedad, es decir, investigar y comparar sobre él: número de acciones de grupos, con visión de largo plazo, por año; número reuniones efectivas, por año del
Consejo Territorial de planeación local; número de organizaciones de participación en procesos de desarrollo territorial, activas por año. Además, es necesario continuar con investigaciones que demuestren la importancia del componente ambiental en la gestión territorial y como sugieren Posada Arrubla et al. (2017), incluir el esfuerzo de la cooperación financiera, para adelantar proyectos subregionales. de manera asociada entre municipios. que cuentan con condiciones naturales similares. Lo anterior. se podría complementar con el aporte de Maltseva (2016), quien propone un sistema regional. para detectar desviaciones y desequilibrios en la gestión. para el desarrollo sostenible del territorio.

Con relación a los objetivos pretendidos y con las prioridades en cada ente territorial analizado, se observa que, para todos, la inclusión del componente ambiental lentamente está dejando de ser una promesa. Este hecho aún no es destacado, debido a que falta concretar lo que significa prioridad para el beneficio colectivo y porque aún es débil la intención administrativa por destinar más recursos en función del entorno natural. Se resalta, que cada vez se asume con mayor seriedad el instrumento Programa de Ejecución y en su interior, el componente ambiental, independiente de que su realización se resuelva en el largo plazo, lo cual, les exige a los gobernantes de turno, dar continuidad a las propuestas anteriores y así sucesivamente.

Como conclusión, en primera instancia, a nivel conceptual, es necesario marcar las diferencias que, hasta ahora, se han generado respecto al uso del término gestión territorial. En especial, porque en la Unión Europea, los análisis sobre el desarrollo territorial parten de la premisa, de que ya existe un desarrollo y que éste se debe cualificar, aplicado en unidades espaciales de tipo regional, mientras que, en América Latina, los países en vía de desarrollo, están aún tratando de concretar sus modelos de desarrollo territorial. Esta diferencia, se evidencia en las pretensiones de la cohesión territorial, que busca reducir desequilibrios entre lugares y, según esta investigación, lo que busca la coherencia de la gestión territorial, es reducir la diferencia en tiempo, en integralidad y en participación, entre lo que se promete y lo que realmente se realiza, a nivel local.

Respecto a la manera de llevar a cabo la gestión territorial en Colombia, no hay homogeneidad, debido a la diferencia socio-cultural, a las características geográficas, a los estilos de administración pública y, sobre todo, al acto o forma de cultura colectiva, que permita llevar a cabo control y mejoras en los procesos. Concretar los modelos de desarrollo territorial deseados exige tiempo, intención política, recursos financieros y mucha participación comunitaria. Para hacer real la gestión territorial, no basta con elaborar un plan, se requiere la articulación de los instrumentos de desarrollo territorial, para demostrar la capacidad de un territorio, de ser abordado desde diferentes objetivos, con enfoques variados, en escalas que van desde lo local, lo subregional, lo regional, hasta lo internacional y en una gradualidad temporal, que permita visualizar una intensión de desarrollo multiespacial, multitemporal y multiobjetivo.

La coherencia está dada por la COgestión de intervenciones efectivas en el territorio, que logren armonía sociedad-naturaleza, 
pero el punto de partida más importante, es la coherencia de cada persona, desde su intención-acción frente al territorio, pues el medio es la gestión colectiva sobre el territorio y el fin, mejorar la calidad de vida colectiva. Nada es imposible y de manera gradual, los entes territoriales pueden ir paulatinamente estableciendo pautas colectivas de respeto, de responsabilidad y de autorregulación, para lograr desarrollo, no solo en el hacer por hacer, sino en todo el ciclo de la gestión como proceso; esto es planearlo bien, hacerlo bien y controlarlo bien. Al final, lo que se busca al darle coherencia a la gestión territorial es la trasformación positiva del desarrollo, desde el conjunto de los grupos sociales. Un asunto es cumplir la norma como parte de una exigencia externa y otra es que, paulatinamente, la sociedad busque, como fundamente de su existencia, el desarrollo armónico de su territorio. Cuando se actúa por convicción, por ética, por cultura, en función del desarrollo de todos, se es coherente, pero si las personas y la sociedad hacemos lo mínimo posible, solo porque la norma lo exige, no hay posibilidades de lograr coherencia en la gestión territorial.

Agradecimientos: Se expresa especial agradecimiento a los líderes comunitarios, expertos temáticos y funcionarios de Barranquilla, Bogotá, Cali y Medellín, quienes amablemente colaboraron, disponiendo de su tiempo y conocimiento, para acceder a las entrevistas personalizadas y aportar en esta investigación. Conflicto de Intereses: El manuscrito fue preparado y revisado por la autora, quien declara ser la que originó el artículo y no presenta conflicto de intereses.

\section{REFERENCIAS}

1. ALCALDÍA DE BARRANQUILLA. 2014. Decreto 0212. Por el cual se adopta el plan de ordenamiento territorial del distrito especial, industrial y portuario de Barranquilla 2012-2032. 362p.

2. ALCALDÍA DE MEDELLÍN. 2014. Acuerdo 048. Por medio del cual se adopta la revisión y ajuste de largo plazo del Plan de Ordenamiento Territorial del Municipio de Medellín y se dictan otras disposiciones complementarias. 877p.

3. ALCALDÍA DE SANTIAGO DE CALI. 2014. Acuerdo 0373. Por medio del cual se adopta la revisión ordinaria de contenido de largo plazo del plan de ordenamiento territorial del municipio de Santiago de Cali. 433p.

4. ALCALDÍA MAYOR DE BOGOTÁ D.C. 2013. Decreto 364. Por el cual se modifican excepcionalmente las normas urbanísticas del Plan de Ordenamiento Territorial de Bogotá D. C., adoptado mediante Decreto Distrital 619 de 2000, revisado por el Decreto Distrital 469 de 2003 y compilado por el Decreto Distrital 190 de 2004. 444p.

5. ARANGO FERNÁNDEZ, W.I. 2007. La Eco-poiesis como fundamento de una perspectiva ambiental para la administración. Gest. Ambient. 10(1):67-82.
6. BAUER, M.A.L.; CARRIÓN, R.deS.M. 2016. Conflitos na gestão social do território: uma análise a partir da organização dos ilhéus em Porto Alegre. Cadernos EBAPE. BR. 14(3):821-835. https://doi.org/10.1590/1679-395131559

7. BOISIER, S. 2001. Sociedad del conocimiento, conocimiento social y gestión territorial. INTERAÇÕES Rev. Internal de Desenvolvimento Local. 2(3):9-28.

8. CABEZA-MORALES, I. 2015. Cohesión territorial: organización para reducir los desequilibrios territoriales. Bitácora. 25(1):51-56. https://doi.org/10.15446/bitacora.v1n25.40147

9. CAMPO-ARIAS, A.; OVIEDO, H.C. 2008. Propiedades psicosométricas de una escala: la consistencia interna. Rev. Salud pública. 10(5):831-839.

10. CASTAÑEDA, R. 2013. Coherencia espacial de la luz en el espacio-fase: procedimientos no-paraxiales e implicaciones físicas. Rev. Acad. Colomb. Cienc. 37(142):37-59.

11. COMISIÓN ECONÓMICA PARA AMÉRICA LATINA Y EL CARIBE, CEPAL. 2010. La hora de la igualdad: Brechas por cerrar, caminos por abrir. Naciones Unidas. 291p.

12. CORTES-TAMAYO, J.O. 1992. Estados coherentes del campo de radiación. Rev. Mexicana de Física. 38(2):309-331.

13. DALLA-TORRE, M.A. 2017. Gobernanza territorial y los planes de ordenamiento territorial: el caso de la provincia Mendoza, Argentina. Bitácora Urbano territorial. 27(1):4754.

https://doi.org/10.15446/bitacora.v27n1.47597

14. DICCIONARIO ETIMOLÓGICO DE CHILE. 2015. Coherencia. Disponible desde Internet en: http:/ / etimologias.dechile.net/?coherencia (con acceso 19/02/2018).

15. DOMÍNGUEZ, M.A. 2009. Análisis de la coherencia en los textos producidos en clases de Física en el nivel polimodal. Rev. Ibero Americana de Educación. 48(3):1-11. https://doi.org/10.35362/rie4832206

16. ESPINOSA CUERVO, J.O. 2006. Ordenamiento Territorial: un instrumento de gestión para el desarrollo sostenible en un ambiente asociativo de concertación entre entidades territoriales. Planeación \& Desarrollo 37(2):111-129.

17. FARINÓS DASÍ, J. 2008. Gobernanza territorial para el desarrollo sostenible: estado de la cuestión y agenda. Boletín de la A.G.E. 46:11-32. 
18. GALÁN-ZAZO, J.I.; SÁNCHEZ-BUENO, M.J. 2004. La relación entre la estrategia y la estructura: un estudio de la coherencia en el contexto español. Decisiones estratégicas, 506-516.

19. GARCÍA L, R.; GARCÍA M, M. 2010. La gestión para resultados en el desarrollo Avances y desafíos en América Latina y El Caribe. BID. 240p.

20. GARCÍA-AYLLÓN, S.; MILLARES, J.L. 2015. New strategies to improve governance in territorial management: evolving from "smart cities" to "smart territories". Procedia Engineering, 118:3-11.

https://doi.org/10.1016/j.proeng.2015.08.396

21. GARETH, A. 2014. What "Is" Territorial Cohesion? What Does It "Do"?: Essentialist Versus Pragmatic Approaches to Using Concepts. European Planning Studies, 22(10):21342155. https://doi.org/10.1080/09654313.2013.819838

22. GÓMEZ SAL, A. 2007. Componentes del valor del paisaje mediterráneo y el flujo de servicios de ecosistemas. Ecosistemas 16(3):97-108.

23. GONZÁLEZ FUENZALIDA, L.E. 2011. Gestión del territorio: un método para la intervención territorial. Universidad de Chile. 93p.

24. LÓPEZ TRIGAL, L. 2015. Diccionario de geografía aplicada y profesional. Disponible desde Internet en: https://www.uv.es/ javier/index_archivos/Diccionario_ Geografia\%20Aplicada.pdf

25. MALTSEVA, A. 2016. System of dynamic norms as a basis for sustainable development management of territories of innovative development. J. Global Entrepreneurship Research. 6(2):1-27. https:/ /doi.org/10.1186/s40497-016-0048-9

26. MASSIRIS CABEZAS, A. 2012. Gestión territorial y desarrollo. Hacia una política de desarrollo territorial sostenible en América Latina. Editorial U. Pedagógica y Tecnológica Colombia. 134p.

27. MÁTTAR, J.; CUERVO, L.M. 2017. Planificación para el desarrollo en América Latina y el Caribe. CEPAL. Chile. 225p.

28. MILLÁN ACEVEDO, N. 2014. Una propuesta metodológica para analizar la coherencia de políticas para el desarrollo. Política Y Sociedad, 51(3):673-693.

https://doi.org/10.5209/rev_POSO.2014.v51.n3.43921

29. MINISTERIO DE AMBIENTE, VIVIENDA Y DESARROLLO TERRITORIAL, MAVDT. 2008. Política de gestión ambiental urbana. Colombia. 52p.
30. MOLINA OCHOA, A. 2010. Un argumento en contra de la coherencia. Revista Diálogos de Saberes. 193-217.

31. MONDRAGÓN RIVERA, C. 2018. Gestión territorial: el tema pendiente en la planificación territorial en Honduras y Centroamérica. Ciencias Espaciales. 10(2):5-23. https://doi.org/10.5377/ce.v10i2.5894

32. MOREA, J.P. 2020. Modelos de desarrollo y soberanía en América Latina: una visión desde la gestión territorial. Geopolíticas, 11(1):71-93. https://dx.doi.org/10.5209/geop.61993

33. MOSTERÍN, J. 2003. El espejo roto del conocimiento y el ideal de una visión coherente del mundo. Rev. Iberoam. Cienc. Tecnol. Soc. 1(1):1-13.

34. PALACIOS-ESPINOSA, X.; RESTREPO-ESPINOSA, M.H. 2008. Aspectos conceptuales e históricos del sentido de coherencia propuesto por antonovsky: ¿una alternativa para abordar el tema de la salud mental? Informes Psicológicos. 10(11):275-300.

35. POSADA ARRUBLA, A.; ESTEBAN CAMPUZANO, A.; BERROCAL MENDOZA, A.E. 2017. Gestión territorial mediante estrategia de asociatividad para Casanare Colombia. Rev. U.D.C.A Act. \& Div. Cient. 20(2):445-456. https://doi.org/10.31910/rudca.v20.n2.2017.382

36. POSADA ARRUBLA, A.; SALAMANCA GARCÍA, J.; GANTIVA, D. 2020. Metodología para relacionar la planeación territorial con el proceso de desertificación. Una aplicación en Colombia. Rev. U.D.C.A Act. \& Div. Cient. 23(1):e1486. https://doi.org/10.31910/rudca.v23.n1.2020.1486

37. POSADA, A. 2014. De la planeación a la gestión ambiental colectiva: posibilidades en Bogotá-Colombia. Rev. U.D.C.A Act. \& Div. Cient. 17(1):257-264. https://doi.org/10.31910/rudca.v17.n1.2014.961

38. REAL ACADEMIA ESPAÑOLA, RAE. 2014. Diccionario de la lengua española. 23. a edición. Disponible desde Internet en:

http:/ / www.rae.es/diccionario-de-la-lengua-espanola/la23a-edicion-2014\#sthash.AQHCfVxB.dpuf

39. RINAUDO R, U.M. 2004. Gestión del desarrollo territorial. Editorial Universidad Piloto de Colombia. 215p.

40. RIVERA PABÓN, J.A. 2013. Debates contemporáneos sobre la planificación territorial y la gestión urbana. Universidad Nacional de Colombia. Luna Azul. 36:307-347. 
41. RODILLA, M.A. 2009. ¿Unidad lógica o dinámica? coherencia y sistema jurídico en Kelsen. DOXA, Cuadernos de filosofía del derecho. 32:255-314.

https://doi.org/10.14198/DOXA2009.32.13

42. SÁNCHEZ-ZAMORA, P.; GALLARDO-COBOS, R.; ROMERO-HUERTAS, C. 2017. Assessing the determinants of territorial cohesion: Evidence from
Colombian departments. Geoforum. 87:48-61.

https://doi.org/10.1016/j.geoforum.2017.10.004

43. VILARNOVO, A. 1990. Coherencia textual: ¿coherencia interna o coherencia externa? ELUA. Estudios de Lingüística. 6:229-239.

https://doi.org/10.14198/ELUA1990.6.12 